\title{
RATIONAL APPROXIMATION ON THE UNION OF SETS
}

\author{
A. M. DAVIE ${ }^{1}$ AND B. K. ØKSENDAL ${ }^{2}$
}

ABstract. A counterexample is given to a conjecture of Val'skir that if $K$ is a compact plane set with interior $U$ and the continuous function $f$ on $K$ satisfies $f \mid \bar{U} \in R(\bar{U})$ and $f \mid b K \in R(b K)$ then $f \in R(K)$. The conjecture is shown to be true when $U$ is a disc.

Let $K$ be a compact subset of the complex plane $C$. We denote by $C(K)$ the algebra of all continuous complex-valued functions on $K$, by $R(K)$ the subalgebra of all uniform limits on $K$ of rational functions with poles outside $K$, and by $A(K)$ the algebra of all functions in $C(K)$ which are analytic on the interior of $K$. In [2] Val'skil conjectured the following: Suppose $U$ is the interior of $K$, and $f \in C(K)$ satisfies $f \mid \bar{U} \in R(\bar{U})$ and $f \mid b K \in R(b K)$. ( $\bar{U}$ or $\mathrm{cl}(U)$ denote the closure of $U, b K$ the boundary of $K$.) Then $f \in R(K)$. He also mentioned the special case when $U$ is the open unit disc $\Delta$. In the present paper a counterexample is given to the general conjecture but it is shown to be true in the case of the disc.

THEOREM 1. There exists a compact set $K$ with interior $U$ and $f \in C(K)$ such that $f|\bar{U} \in R(\bar{U}), f| b K \in R(b K)$, but $f \notin R(K)$.

Proof. Let $\Gamma$ be an arc with endpoints -1 and 1 lying in $\Delta$, with positive continuous analytic capacity $[1$, p. 203$] . \Delta \backslash \Gamma$ has two components $U$ and $V$ say. Let $\left\{\Delta_{j}\right\}_{j=1}^{\infty}$ be a sequence of disjoint open discs in $V$ such that $V \backslash \bigcup_{j=1}^{\infty} \Delta_{j}$ is nowhere dense, and the sum of the radii of the discs is finite. Let $K=\bar{\Delta} \backslash \bigcup_{j=1}^{\infty} \Delta_{j}$. Then the interior of $K$ is $U$ and $b K=b \Delta \cup \Gamma \cup \operatorname{cl}\left(\bigcup_{j=1}^{\infty} b \Delta_{j}\right)$. Since $\alpha(\Gamma)>0$, there exists an $f$ continuous on $\boldsymbol{C}$ and analytic outside $\Gamma$ such that $f^{\prime}(\infty) \neq 0$. Let $\mu$ be the measure on $b K$ which coincides with $d z$ on $b \Delta$, with $-d z$ on $\bigcup_{j=1}^{\infty} b \Delta_{j}$ and is zero on the rest of $b K$. Then $\int g d \mu=0$ for every rational function with poles outside $K$ and hence $\int g d \mu=0$ for all $g \in R(K)$. Since $\int_{b K} f d \mu=\int_{b \Delta} f d z=2 \pi i \cdot f^{\prime}(\infty) \neq 0, f \mid K \notin R(K)$. Since $\bar{U}$ has connected complement, $R(\bar{U})=A(\bar{U})$ and so $f \mid \bar{U} \in R(\bar{U})$. Likewise $C \backslash(\bar{V} \cup b U)$ has just two components, so $R(\bar{V} \cup b U)$

Received by the editors October 23, 1970.

AMS 1969 subject classifications. Primary 3070.

Key words and phrases. Compact plane set, rational approximation, $T_{\varphi}$ operator, analytically negligible set.

1 This work was partially supported by NSF Grant GP-19067.

2 This work was partially supported by NSF Grant GP-11475. 
$=A(\bar{V} \cup b U)$ and hence $f \mid \bar{V} \cup b U \in R(\bar{V} \cup b U)$. Since $b K \subset \bar{V} \cup b U$, it follows that $f \mid b K \in R(b K)$.

Before proving the second theorem we make some remarks about the $T_{\varphi}$ operator. Let $\varphi$ be a $C^{1}$ function with compact support and $f$ be a continuous function on $C$. We define $T_{\varphi} f$ by

$$
T_{\varphi} f(\zeta)=\varphi(\zeta) f(\zeta)+\frac{1}{\pi} \cdot \int \frac{f(z)}{z-\zeta} \cdot \frac{\partial \varphi}{\partial \bar{z}} d m(z), \quad \zeta \in C,
$$

where $m$ denotes plane Lebesgue measure. Then $T_{\varphi} f$ is continuous where $f$ is, analytic outside the support of $\varphi$ and on any open set where $f$ is analytic. See for example [1, p. 29].

THEOREM 2. Let $Q \subseteq K$ be compact. Let $f \in C(K)$ such that each $z \in K \backslash Q$ has a neighbourhood $V$ with $f \mid \operatorname{cl}(K \cap V) \in R(\operatorname{cl}(K \cap V))$. Let $\epsilon>0$. Then there exists $g$, continuous on $\mathbf{C}$ and analytic on a neighbourhood of $K \backslash Q$, with $|f-g|<\epsilon$ on $K$.

Proof. Extend $f$ to be continuous on $C$ with compact support. Let $M_{n}=\left\{z \in K ; d(z, Q) \geqq 2^{-n}\right\}, n=1,2, \cdots$. Then $M_{n}$ is compact and $K \backslash Q=\cup_{n} M_{n}$. We construct by induction on $n$ sequences $\left\{W_{n}\right\}$, $\left\{f_{n}\right\}$, where $W_{n}$ is a compact neighbourhood of $M_{n}, W_{n} \subseteq W_{n+1}$, and $f_{n}$ is continuous on $\boldsymbol{C}$ with compact support and analytic on a neighbourhood of $W_{n}$ and moreover each $z \in K \backslash Q$ has a neighbourhood $V$ with $f_{n} \mid \operatorname{cl}(K \cap V) \in R(\operatorname{cl}(K \cap V)),\left\|f_{1}-f\right\|<\epsilon / 2$ and $\left\|f_{n+1}-f_{n}\right\|$ $<\epsilon / 2^{n+1}$ for all $n$.

Inductive step (initial step similar). Suppose $f_{n}, W_{n}$ constructed. Let $U$ be a neighbourhood of $W_{n}$ on which $f_{n}$ is analytic. Let $\Delta_{1}, \cdots, \Delta_{r}$ be a covering of $M_{n+1}$ by open discs such that $f_{n} \mid \operatorname{cl}\left(K \cap \Delta_{k}\right) \in R\left(\operatorname{cl}\left(K \cap \Delta_{k}\right)\right)$ for each $k$ and such that any $\Delta_{k}$ which meets $W_{n}$ must lie in $U$. Let $\varphi_{1}, \cdots, \varphi_{r}$ be $C^{1}$ functions, $\varphi_{k}$ supported on a compact subset of $\Delta_{k}$, with $\sum_{k=1}^{r} \varphi_{k}=1$ on a neighbourhood of $M_{n+1}$. Fix $k$; we can find a sequence $\left\{g_{s}\right\}, g_{s}$ continuous on $\boldsymbol{C}$ and analytic on a neighbourhood of $\operatorname{cl}\left(K \cap \Delta_{k}\right), g_{s} \rightarrow f_{n}$ uniformly. Then $T_{\varphi_{k}} g_{s} \rightarrow T_{\varphi_{k}} f_{n}$ uniformly; moreover $T_{\varphi_{k}} g_{\mathrm{s}}$ is analytic in a neighbourhood of $K$. For each $k$ we define $F_{k}$ as follows: If $\Delta_{k}$ meets $W_{n}$, put $F_{k}=0$ (in this case $T_{\varphi_{k}} f_{n}=0$ ). If $\Delta_{k}$ does not meet $W_{n}$ choose $g_{s}$ such that

$$
\left\|T_{\varphi_{k} g_{s}}-T_{\varphi_{k}} f_{n}\right\|<\epsilon /\left(2^{n+1} \cdot r\right)
$$

and put $F_{k}=T_{\varphi_{k}} g_{\imath}$. Then $F_{k}$ is analytic in a neighbourhood of $K \cup W_{n}$. Put

$$
f_{n+1}=f_{n}-\sum_{k=1}^{r} T_{\varphi_{k}} f_{n}+\sum_{k=1}^{r} F_{k}
$$


Since $\sum_{k=1}^{r} \varphi_{k}=1$ on a neighbourhood of $M_{n+1}, f_{n}-\sum_{k=1}^{r} T_{\varphi_{k}} f_{n}$ is analytic in a neighbourhood of $M_{n+1}$. Also it is analytic on a neighbourhood of $W_{n}$ since $f_{n}$ is. Hence $f_{n+1}$ is analytic on a neighbourhood of $M_{n+1} \cup W_{n}$. Let $W_{n+1}$ be a compact neighbourhood of $W_{n} \cup M_{n+1}$ such that $f_{n+1}$ is analytic on a neighbourhood of $W_{n+1}$. That each $z \in K \backslash Q$ has a neighbourhood $V$ with $f_{n+1} \mid \operatorname{cl}(K \cap V) \in R(\operatorname{cl}(K \cap V))$ follows from the fact that each $T_{\varphi_{k}} f_{n}$ and each $F_{k}$ has this property. Finally

$$
\left\|f_{n+1}-f_{n}\right\| \leqq \sum_{k=1}^{r}\left\|F_{k}-T_{\varphi_{k}} f_{n}\right\|<\epsilon / 2^{n+1}
$$

This completes the induction.

We have $f_{n} \rightarrow g$ say, uniformly, where $g$ is analytic on the interior of $W_{n}$ for each $n$; hence on a neighbourhood of $U_{n} M_{n}=K \backslash Q$. Finally

$$
\|f-g\| \leqq\left\|f-f_{1}\right\|+\sum_{n=1}^{\infty}\left\|f_{n+1}-f_{n}\right\|<\epsilon,
$$

and the proof is complete.

A compact set $E$ is analytically negligible if every continuous function on $\boldsymbol{C}$ which is analytic on an open set $V$ can be approximated uniformly on $\boldsymbol{C}$ by functions continuous on $\boldsymbol{C}$ and analytic on $V \cup E$. Since a circle is analytically negligible $[1,8.12 .3]$, the following result answers Val'skir's question affirmatively when $U$ is a disc:

COROLlaRY. Let $K, L$ be a compact sets such that $b K \cap b L$ is analytically negligible. Let $f \in C(K \cup L)$ such that $f \mid K \in R(K)$ and $f \mid L \in R(L)$. Then $f \in R(K \cup L)$.

Proof. Theorem 2 applies with $K \cup L$ in place of $K$ and $Q=b K$ $\cap b L$. Given $\epsilon>0$, we can find $g$ continuous on $C$ and analytic on a neighbourhood $U$ of $K \cup L \backslash(b K \cap b L)$ with $|f-g|<\epsilon / 2$ on $K$. Since $b K \cap b L$ is analytically negligible, we can find $h$, continuous on $C$ and analytic on $U$ and on a neighbourhood of $b K \cap b L,\|h-g\|<\epsilon / 2$. Then $h$ is analytic on a neighbourhood of $K \cup L$ and $|f-h|<\epsilon$ on $K$. Hence $f \in R(K \cup L)$.

Some open problems. The following questions are closely related to the contents of this paper.

(1) Suppose $f \in C(K)$ and that for each $z \in K$ where $f$ does not vanish we can find a neighbourhood $V$ of $z$ such that $f \mid(\bar{V} \cap K)$ $\in R(\bar{V} \cap K)$.

Must $f \in R(K)$ ?

Theorem 2 shows that the answer is yes provided the zero set of 
$f$ is analytically negligible. An affirmative answer would also settle the following question due to Björk (private communication).

(2) Suppose $f \in C(K)$ and $f^{2} \in R(K)$. Must $f \in R(K)$ ?

The final question is a somewhat weaker version of (1) which we have nevertheless been unable to prove.

(3) Suppose $f \in C(K \cup L)$ satisfies $f \mid K \in R(K)$ and $f=0$ on $L$. Must $f \in R(K \cup L)$ ?

\section{REFERENCES}

1. T. W. Gamelin, Uniform algebras, Prentice-Hall, Englewood Cliffs, N. J., 1969.

2. R. E. Val'skir, On parts of algebras of analytic functions and measures orthogonal to these algebras, Sibirsk. Math. Ž. 8 (1967), 1222-1235=Siberian Math. J. 8 (1967), 935-944. MR 36 \#1986.

University of California, Los Angeles, California 90024 